\title{
Economic Evaluations of Gestational Diabetes Mellitus Screening: A Systematic Review
}

\author{
Xiuting Mo ${ }^{1,2}$, Ruoyan Gai Tobe ${ }^{1,3}$, Yoshimitsu Takahashi ${ }^{2}$, Naoko Arata ${ }^{4}$, \\ Tippawan Liabsuetrakul ${ }^{5}$, Takeo Nakayama ${ }^{2}$, and Rintaro Mori ${ }^{1}$ \\ ${ }^{1}$ Department of Health Policy, National Center for Child Health and Development, Tokyo, Japan \\ ${ }^{2}$ Department of Health Informatics, Kyoto University School of Public Health, Kyoto, Japan \\ ${ }^{3}$ Department of Empirical Social Security Research, National Institute of Population and Social Security Research, Tokyo, Japan \\ ${ }^{4}$ Division of Women's Health and Reproductive Endocrinology, National Center for Child Health and Development, Tokyo, Japan \\ ${ }^{5}$ Epidemiology Unit, Prince of Songkla University, Songkla, Thailand
}

Received January 10, 2020; accepted March 22, 2020; released online May 23, 2020

\begin{abstract}
Background: This study aims to find evidence of the cost-effectiveness of gestational diabetes mellitus (GDM) screening and assess the quality of current economic evaluations, which have shown different conclusions with a variation in screening methods, data sources, outcome indicators, and implementation in diverse organizational contexts.

Methods: Embase, Medline, Web of Science, Health Technology Assessment, database, and National Health Service Economic Evaluation Database databases were searched through June 2019. Studies on economic evaluation reporting both cost and health outcomes of GDM screening programs in English language were selected, and the quality of the studies was assessed using Drummond's checklist. The general characteristics, main assumptions, and results of the economic evaluations were summarized.

Results: Our search yielded 10 eligible economic evaluations with different screening strategies compared in different settings and perspectives. The selected papers scored 81\% (68-97\%) on the items in Drummond's checklist on average. In general, a screening program is cost-effective or even dominant over no screening. The one-step screening, with more cases detected, is more likely to be cost-effective than the two-step screening. Universal screening is more likely to be cost-effective than screening targeting the high-risk population. Parameters affecting cost-effectiveness include: diagnosis criteria, epidemiological characteristics of the population, efficacy of screening and treatment, and costs.

Conclusions: Most studies found GDM screening to be cost-effective, though uncertainties remain due to many factors. The quality assessment identified weaknesses in the economic evaluations in terms of integrating existing data, measuring costs and consequences, analyzing perspectives, and adjusting for uncertainties.
\end{abstract}

Key words: gestational diabetes mellitus screening; economic valuation; review

Copyright ( $) 2020$ Xiuting Mo et al. This is an open access article distributed under the terms of Creative Commons Attribution License, which permits unrestricted use, distribution, and reproduction in any medium, provided the original author and source are credited.

\section{INTRODUCTION}

Gestational diabetes mellitus (GDM) is defined as any degree of glucose intolerance with onset or first recognition during pregnancy. Approximately $17.8 \%$ (range, 9.3-25.5\%) of pregnant women suffer complications due to GDM, depending on the epidemiological characteristics of the population investigated and diagnostic tests employed. ${ }^{1}$ GDM has become an important public health issue and is responsible for increased risks of maternal, prenatal, and neonatal complications, such as type 2 diabetes mellitus (T2DM) and cardiovascular disease in mothers and obesity and long-term metabolic syndrome in their offspring, ${ }^{2}$ potentially increasing the economic burden of healthcare. It is possible to manage GDM during pregnancy using nutritional management, insulin treatment, or oral hypoglycemic agent, with the primary goal of maintaining blood glucose within normal levels. Moreover, monitoring and prevention of T2DM in women with prior GDM in the postnatal period is also important in reducing the long-term disease burden. Women with GDM were found to have a higher risk of developing postpartum diabetes. ${ }^{3}$ For the offspring, diabetes, cardiovascular alterations, and/or obesity in adulthood are the lifelong consequences of intrauterine exposure to increased glucose. ${ }^{4,5}$

There are many studies on the economic evaluations of GDM management during both prenatal and postnatal periods. ${ }^{6,7}$ To manage GDM, many countries have implemented a screening program to identify asymptomatic pregnant women. However, the definition of GDM, the target population, and clinical practices vary among studies. ${ }^{8}$ GDM screening protocols are of two types and their modifications: a two-step method (a first-step glucose challenge test [GCT] and a second-step oral glucose tolerance test [OGTT]) that diagnoses based on two or more 
abnormal values $(5.3 \mathrm{mmol} / \mathrm{L}$ while fasting, $10.0 \mathrm{mmol} / \mathrm{L} 1$ hour postprandial, and $8.6 \mathrm{mmol} / \mathrm{L} 2$ hours postprandial) on OGTT and a one-step method that recommends a $75 \mathrm{~g}$ OGTT test without a $50 \mathrm{~g}$ GCT before and has a simpler, one-abnormal-value diagnosis criteria. ${ }^{9}$ From an economic evaluation perspective, different conclusions have been drawn due to the different screening methods; data sources, outcomes, and interventions vary widely across studies examining disparate systems in diverse organizational contexts. Therefore, this study aims to systematically review the evidence on the cost-effectiveness of GDM screening and perform a quality assessment.

\section{METHODS}

\section{Literature search}

We conducted two independent searches of the related literature through June 2019 by Mo and Gai. We searched Embase, Medline, Web of Science, Health Technology Assessment (HTA) database, and National Health Service Economic Evaluation Database (NHSEED) for studies related to "economic evaluation of gestational diabetes screening" using the following search strings in Embase, MEDLINE, Web of Science, and NHSEED: $\mathrm{TS}=\left(\left(\left(\right.\right.\right.$ diabet $^{*}$ AND $\left(\right.$ pregnanc $^{*}$ OR pregnant OR gestation* OR wom?n OR female* OR mother $\left.{ }^{\star}\right)$ ) OR gdm) AND (screening* OR diagnos* OR glucose tolerance*) AND (( cost $^{*}$ AND (effectiveness OR benefit* OR utility)) OR (economic AND evaluation* $\left.\left.{ }^{*}\right)\right)$. In HTA, the search strings used were: (((diabet* and (pregnanc* or pregnant or gestation* or wom?n or female* or mother*)) or gdm) and (screening* or diagnos* or glucose tolerance $\left.{ }^{\star}\right)$ ). We did not select a time range for the search. All citations were imported into EndNote for further screening.

\section{Screening of studies}

The screening was conducted by Mo under the supervision of Gai. The studies were screened in three steps. First, all duplicate papers were found using EndNote; second, all the apparently relevant studies were selected by reviewing their titles and abstracts; and last, the full texts were read. The inclusion criteria were: 1) cost-effectiveness analysis, reporting both input of health resources and output of health gains; 2) studies of screening programs for detecting GDM during pregnancy among women of reproductive age; and 3) original studies involving decision modelling or other mathematical methodologies to deal with uncertainties in cost-effectiveness. The studies that only reported cost or effectiveness and did not discuss the trade-off on marginal costs or health gains were excluded (see PRISMA 2009 Checklist in eTable 1).

\section{Quality assessment and critical appraisal}

We assessed the quality of the included studies using the Assessing Economic Evaluations Checklist from the Methods for the Economic Evaluation of Health Care Programmes, ${ }^{10}$ which contains 10 major questions on the following: answerable question posed; competing alternatives given; effectiveness of the programs or services established; costs and consequences identified; costs and consequences measured accurately, credibly, and adjusted for differential timing; incremental analysis performed; uncertainty characterized; and discussions including all issues of concern to the users. Each question contains several sub-questions. The responses available are: "Yes," "Partially yes," "No," and "Can't tell." A "Yes" is equivalent to a full score, a "No" has a value of 0 , and a "Partially yes" or "Can't tell" has a value of half a point each. For each "Not Applicable" (N.A.) response, the corresponding sub-question is disregarded (eTable 2). The quality of one paper ${ }^{11}$ was independently assessed thrice and the divergences and cases of "Partially yes" and "Can't tell" were fully discussed by Mo, Agari Takahiro, and Naito Yumi. Then, the rest of the evaluation was completed by Mo.

\section{RESULTS}

\section{Study selection}

Embase, MEDLINE, HTA, Web of Science, and NHSEED yielded a total of 136, 104, 30, 317, and 21 articles, respectively. The search results were updated in June 2019. In all, 608 studies were identified. We excluded 93 duplicated studies and 480 articles that did not discuss GDM screening during pregnancy or only covered cost estimates or effectiveness. Fourteen poster or abstract sessions ${ }^{12-25}$ and 10 other types of articles ${ }^{26-35}$ were excluded (see Table 1). Of the remaining 11 articles, two $^{36,37}$ that reported similar results using the same model were considered a single study. Finally, 10 studies ${ }^{11,36-45}$ were included and analyzed (Figure 1).

\section{General characteristics of the economic evaluations} Four of the included studies ${ }^{39,41,42,45}$ used TreeAge and three used Microsoft Excel ${ }^{37,40,43}$ to construct a decision tree for their economic model. Their general characteristics are summarized in Table 2. The first study was published in 2002 by Poncet ${ }^{45}$ and the next one in $2005 .{ }^{44}$ The remaining eight studies were published between 2011 and 2017. ${ }^{11,36-43}$ Four evaluations were from the United States, ${ }^{36,41,42,44}$ three were from Europe (United Kingdom, ${ }^{38}$ Ireland, ${ }^{11}$ and France $\left.^{45}\right)$, one was from New Zealand, ${ }^{40}$ and the remaining two were from Asia (Singapore, ${ }^{39}$ India, and Israel ${ }^{36,37}$ ). Most of the studies used cost-utility analysis (CUA), where utility is measured in quality-adjusted life years (QALYs) or disability-adjusted life years (DALYs). Two studies used cost-effectiveness analysis (CEA)—one considered cases detected as the outcome, ${ }^{40}$ while the other used prevented pregnancy complications like, macrosomia, prematurity, perinatal mortality, and hypertensive disorders as the outcome. ${ }^{45}$ In terms of economic evaluation, five of the publications were from a healthcare perspective (third-party payer), ${ }^{11,38,40,41,43}$ one was from the payers' perspective, ${ }^{39}$ and two were from a societal perspective. ${ }^{42,44}$ The remaining three studies did not clarify their perspective. ${ }^{36,37,45}$ One study was supported by a pharmaceutical company (Novo Nordisk), ${ }^{36,37}$ one failed to mention any funding, ${ }^{42}$ and the others were supported by public funding.

The majority $(8 / 10)$ of the selected studies included "no screening" for comparison. ${ }^{11,36-39,41,44,45}$ Large variations were found in the screening options, with three studies evaluating screening at different coverage rates (universal or high-risk targets), ${ }^{38,39,45}$ while one compared screening in different settings (GP practice or hospital-based). ${ }^{11}$ Two studies projected the longterm impact of screening on diabetes prevention. ${ }^{36,37,41}$ Most studies used the diagnostic criteria of the International Association of Diabetes and Pregnancy Study Groups (IADPSG) released in $2010^{11,36,37,39-42}$ or Carpenter and Coustan $(C C)^{41,45}$; one used the 2008 guidelines of National Institute of Health and Clinical Excellence (NICE) ${ }^{43}$; and one compared different diagnostic thresholds (NICE guidelines of 2015 and IADPSG) ${ }^{38}$ 
Table 1. Characteristics of excluded studies at second-level screening

\begin{tabular}{|c|c|c|}
\hline First author & Year & Reason for exclusion \\
\hline Cade $\mathrm{TJ}^{26}$ & 2019 & Cost and effectiveness were separately discussed \\
\hline Liang $\mathrm{SQ}^{12}$ & 2019 & Only abstract available \\
\hline Duarte $A^{13}$ & 2018 & Only abstract available \\
\hline $\mathrm{Li} \mathrm{LJ}^{14}$ & 2018 & Only abstract available \\
\hline Sortso $\mathrm{C}^{27}$ & 2018 & Only related to cost calculation \\
\hline Rodrigues $^{28}$ & 2017 & Only compared cost per case detected, did not include trade-off in marginal cost or health gain \\
\hline Walker $\mathrm{AR}^{15}$ & 2017 & Only abstract available \\
\hline Pearson $\mathrm{LJ}^{16}$ & 2016 & Only abstract available \\
\hline Ming WK ${ }^{17}$ & 2016 & Only abstract available \\
\hline Zhang $\mathrm{L}^{18}$ & 2015 & Only abstract available \\
\hline Quitian $\mathrm{H}^{19}$ & 2015 & Only abstract available \\
\hline Duran $A^{29}$ & 2014 & Only compared cost saving per case, did not include trade-off in marginal cost or health gain \\
\hline Chen PY 20 & 2014 & Only abstract available \\
\hline Gillespie $^{30}$ & 2012 & Only related to cost per case detected \\
\hline Werner $\mathrm{EF}^{21}$ & 2012 & Only abstract available \\
\hline Reel $\mathrm{M}^{22}$ & 2011 & Only abstract available \\
\hline Van Leeuwen $\mathrm{M}^{23}$ & 2009 & Only abstract available \\
\hline Lee $S^{24}$ & 2008 & Only abstract available \\
\hline Thung $\mathrm{S}^{25}$ & 2007 & Only abstract available \\
\hline Ayach $\mathrm{W}^{31}$ & 2006 & Cost and effectiveness were separately discussed \\
\hline Rey $E^{32}$ & 2004 & Cost and effectiveness were separately discussed \\
\hline Larijani $\mathrm{B}^{33}$ & 2004 & Only related to cost per case screened/detected \\
\hline Di Cianni $\mathrm{G}^{34}$ & 2002 & Only related to cost per case detected \\
\hline Weiner $\mathrm{CP}^{35}$ & 1986 & Only related to cost per case identified \\
\hline
\end{tabular}

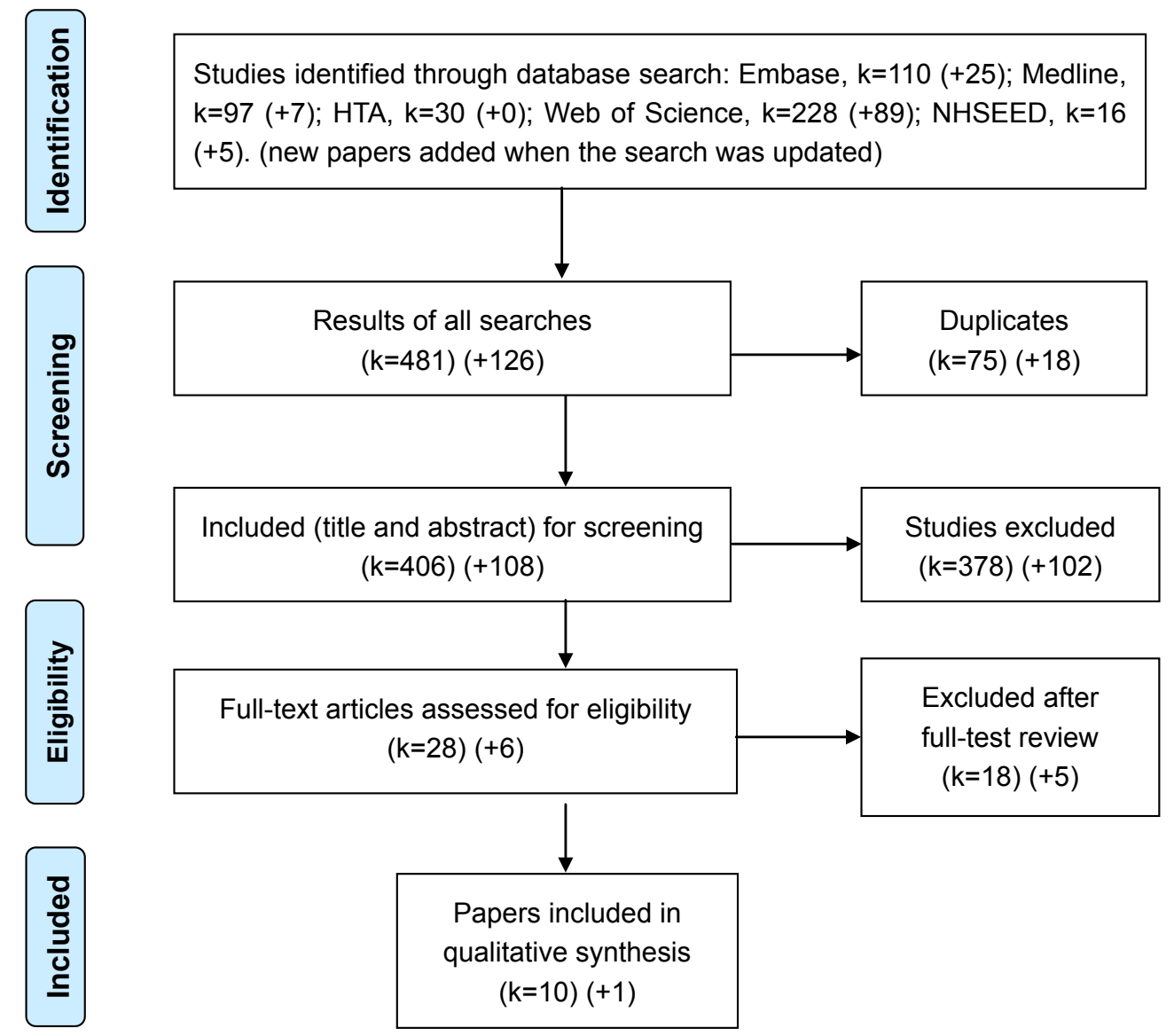

Figure 1. Items selected for systematic review and meta-analyses (PRISMA). Embase, Excerpta Medica Database; HTA, Health Technology Assessment; Medline, Medical Literature Analysis and Retrieval System Online; NHSEED, National Health Service Economic Evaluation Database. The search was updated in June 2019. 
Mo X, et al.

Table 2. Study overview of published economic evaluations of GDM screening

\begin{tabular}{|c|c|c|c|c|c|c|}
\hline $\begin{array}{l}\text { First authors, } \\
\text { year }\end{array}$ & $\begin{array}{l}\text { Country/ } \\
\text { population }\end{array}$ & Journal & $\begin{array}{l}\text { Type of } \\
\text { economic } \\
\text { evaluation }\end{array}$ & Perspective & Funding & Comparators (criteria/cutoff value) \\
\hline $\begin{array}{l}\text { Jacklin PB, } \\
2017\end{array}$ & UK & BMJ & $\begin{array}{l}\text { CUA } \\
(\mathrm{QALY})\end{array}$ & Healthcare & $\begin{array}{l}\text { the National Institute } \\
\text { for Health and Care } \\
\text { Excellence }\end{array}$ & $\begin{array}{l}\text { 1. no screening; 2. NICE } 2015 \text { diagnostic } \\
\text { threshold 3. IADPSG threshold. (universal } \\
\text { screening on baseline; population with or } \\
\text { without risk factors for subgroup analysis) }\end{array}$ \\
\hline $\begin{array}{l}\text { Danyliv A, } \\
2016\end{array}$ & Ireland & Diabetologia & $\begin{array}{l}\text { CUA } \\
(\mathrm{QALY})\end{array}$ & Healthcare & $\begin{array}{l}\text { the Health Research } \\
\text { Board of Ireland }\end{array}$ & $\begin{array}{l}\text { 1. no screening; } 2 \text {. universal } 2 \mathrm{~h} 75 \mathrm{~g} \text { OGTT } \\
\text { (IADPSG) at a GP practice; } 3 \text {. universal } 2 \mathrm{~h} \\
75 \mathrm{~g} \text { OGTT (IADPSG) at hospital-based } \\
\text { screening }\end{array}$ \\
\hline $\begin{array}{l}\text { Chen PY, } \\
2016\end{array}$ & Singapore & $\begin{array}{l}\text { Asia Pac J } \\
\text { Public Health }\end{array}$ & $\begin{array}{l}\text { CUA } \\
(\text { QALY) }\end{array}$ & Payers & $\begin{array}{l}\text { Health Services and } \\
\text { Systems Research } \\
\text { Program at Duke-NUS } \\
\text { Graduate Medical School } \\
\text { and KK women's and } \\
\text { Children's hospital from } \\
\text { Singhealth Group }\end{array}$ & $\begin{array}{l}\text { 1. no screening; 2. universal } 2 \mathrm{~h} 75 \mathrm{~g} \text { OGTT } \\
\text { (IADPSG); 3. targeted } 2 \mathrm{~h} 75 \mathrm{~g} \text { OGTT } \\
\text { (IADPSG) based on risk factors following } \\
\text { NICE guidelines }\end{array}$ \\
\hline $\begin{array}{l}\text { Coop C, } \\
2015\end{array}$ & New Zealand & BMJ & CEA & Healthcare & $\begin{array}{l}\text { the Ministry of Health to } \\
\text { support the development } \\
\text { of a clinical practice } \\
\text { guideline }\end{array}$ & $\begin{array}{l}\text { 1. at first booking HbA1c test }+ \text { at } \\
\text { 24-28 weeks (IADPSG) } 2 \mathrm{~h} 75 \mathrm{~g} \text { OGTT; } \\
\text { 2. at first booking HbA1c test }+ \text { at } \\
\text { 24-28 weeks } 1 \mathrm{~h} 50 \mathrm{~g} \mathrm{GCT} \pm 2 \mathrm{~h} 75 \mathrm{~g} \\
\text { OGTT (IADPSG) }\end{array}$ \\
\hline $\begin{array}{l}\text { Werner EF, } \\
2012\end{array}$ & US & Diabetes Care & $\begin{array}{l}\text { CUA } \\
\text { (QALY) }\end{array}$ & Healthcare & $\begin{array}{l}\text { Department of Obstetrics, } \\
\text { Gynecology, and } \\
\text { Reproductive Sciences } \\
\text { at the Yale School of } \\
\text { Medicine }\end{array}$ & $\begin{array}{l}\text { 1. no screening; } 2 \text {. at } 24-28 \text { weeks } 1 \mathrm{~h} 50 \mathrm{~g} \\
\text { GCT } \pm 3 \mathrm{~h} 100 \mathrm{~g} \text { OGTT (Carpenter and } \\
\text { Coustan criteria); } 3 \text {. at first booking } \\
\text { FBG } \pm \text { at } 24-28 \text { weeks } 2 \mathrm{~h} 75 \mathrm{~g} \\
\text { OGTT (IADPSG) }\end{array}$ \\
\hline $\begin{array}{l}\text { Mission JF, } \\
2012\end{array}$ & US & $\begin{array}{l}\text { Am J Obstet } \\
\text { Gynecol }\end{array}$ & $\begin{array}{l}\text { CUA } \\
\text { (QALY) }\end{array}$ & societal & not mentioned & $\begin{array}{l}\text { 1. at } 24-28 \text { weeks } 1 \mathrm{~h} 50 \mathrm{~h} \mathrm{GCT} \pm 3 \mathrm{~h} 100 \mathrm{~g} \\
\text { OGTT (Carpenter and Coustan criteria); } \\
\text { 2. } 2 \mathrm{~h} 75 \mathrm{~g} \text { OGTT (IADPSG) }\end{array}$ \\
\hline $\begin{array}{l}\text { Lohse N, } \\
2011 \text { (Marseille E, } \\
\text { 2013) }\end{array}$ & India and Israel & $\begin{array}{l}\text { Int J Gynaecol } \\
\text { Obstet (J } \\
\text { Matern Fetal } \\
\text { Neonatal } \\
\text { Med) }\end{array}$ & $\begin{array}{l}\text { CUA } \\
\text { (DALY) }\end{array}$ & $\begin{array}{l}\text { not } \\
\text { mentioned }\end{array}$ & Novo Nordisk A/S & $\begin{array}{l}\text { 1. no screening; } 2.2 \mathrm{~h} 75 \mathrm{~g} \text { OGTT and } \\
\text { followed by prenatal intervention and } \\
\text { postpartum preventive lifestyle prevention } \\
\text { (IADPSG) }\end{array}$ \\
\hline $\begin{array}{l}\text { Round JA, } \\
2011\end{array}$ & UK & Diabetologia & $\begin{array}{l}\text { CUA } \\
(\text { QALY) }\end{array}$ & Healthcare & $\begin{array}{l}\text { National Health Service } \\
\text { in England and Wales }\end{array}$ & $\begin{array}{l}\text { 1. no screening; 2. } 2 \mathrm{~h} 75 \mathrm{~g} \text { OGTT; } 3 \text {. FPG; } \\
\text { 4. RBG; } 5 \text {. GCT; } 6 . \mathrm{RBG} \pm 2 \mathrm{~h} 75 \mathrm{~g} \text { OGTT; } \\
\text { 7. FPG } \pm 2 \mathrm{~h} 75 \mathrm{~g} \text { OGTT; } 8 \text {. GCT } \pm 2 \mathrm{~h} 75 \mathrm{~g} \\
\text { OGTT. (NICE guidance, 2008) }\end{array}$ \\
\hline $\begin{array}{l}\text { Nicholson WK, } \\
2005\end{array}$ & US & Diabetes Care & $\begin{array}{l}\text { CUA } \\
\text { (QALY) }\end{array}$ & societal & $\begin{array}{l}\text { Robert Wood Johnson } \\
\text { Foundation and the } \\
\text { National Institute of } \\
\text { Diabetes and Digestive } \\
\text { and Kidney Diseases }\end{array}$ & $\begin{array}{l}\text { 1. no screening; } 2.1 \mathrm{~h} 50 \mathrm{~g} \text { GCT } \\
(140 \mathrm{gm} / \mathrm{dl}) \pm 3 \mathrm{~h} 100 \mathrm{~g} \mathrm{GTT} ; 3.2 \mathrm{~h} 75 \mathrm{~g} \\
\text { GTT }(\geq 140 \mathrm{gm} / \mathrm{dl}) ; 4.3 \mathrm{~h} 100 \mathrm{~g} \mathrm{GTT} \\
(95 / 180 / 155 / 145 \mathrm{mg} / \mathrm{dl}(0 / 1 / 2 / 3 \mathrm{~h}))\end{array}$ \\
\hline $\begin{array}{l}\text { Poncet B, } \\
2002\end{array}$ & France & $\begin{array}{l}\text { Eur J Obstet } \\
\text { Gynecol } \\
\text { Reprod Biol }\end{array}$ & CEA & $\begin{array}{l}\text { not } \\
\text { mentioned }\end{array}$ & Health ministry & $\begin{array}{l}\text { 1. no screening; } 2 \text {. screening of high-risk } \\
\text { women with } 1 \mathrm{~h} 50 \mathrm{~g} \mathrm{GCT} \pm 3 \mathrm{~h} 100 \mathrm{~g} \\
\text { OGTT (Carpenter and Coustan criteria); } \\
\text { 3. screening of all women with } 1 \mathrm{~h} 50 \mathrm{~h} \\
\text { GCT } \pm 3 \mathrm{~h} 100 \mathrm{~g} \text { OGTT (Carpenter } \\
\text { and Coustan criteria); } 4 \text {. screening of all } \\
\text { women according to } 2 \mathrm{~h} 75 \mathrm{~g} \text { OGTT. } \\
(\geq 5.5 / 8 \mathrm{mmol} / 1(0 / 2 \mathrm{~h}))\end{array}$ \\
\hline
\end{tabular}

Carpenter and Coustan criteria (1998), A $1 \mathrm{~h}$ glucose value $\geq 7.2 \mathrm{mmol} / 1$ indicates the need for a $100 \mathrm{~g}$ OGTT, and a diagnosis of GDM is made if in the fasting state: $\geq 5.3,10.0,8.6$ or $7.8 \mathrm{mmol} / \mathrm{L}(0 / 1 / 2 / 3 \mathrm{~h})$; CEA, cost effectiveness analysis; CUA, cost utility analysis; DALY, disability adjusted of life year; FPG, fasting plasma glucose; GCT, glucose challenge test; GDM, gestational diabetes mellitus; HbA1c test, haemoglobin A1c or glycated haemoglobin test; NICE, the National Institute for Health and Care Excellence; NICE 2015 threshold, GDM is defined as a FBG $\geq 5.6 \mathrm{mmol} / \mathrm{L}, 2 \mathrm{~h} 75 \mathrm{~g}$ OGTT $\geq 7.8 \mathrm{mmol} / \mathrm{L}$; IADPSG, International Association of the Diabetes and Pregnancy Study Groups. FPG $\geq 5.1 \mathrm{mmol} / \mathrm{L}$, a $75 \mathrm{~g}$ 1-hour OGTT $\geq 10.0 \mathrm{mmol} / \mathrm{L}$, or a $75 \mathrm{~g} 2$-hour OGTT $\geq 8.5 \mathrm{mmol} / \mathrm{L}$; (O)GTT, (oral) glucose tolerance test; QALY, quality adjusted life year; RBG, random blood glucose.

\section{Main assumptions and results of economic evalua- tions}

The major findings and sensitivity analysis results are summarized in Table 3, and the detailed input parameters of each study are presented in eTable 3 and eTable 4. As an important parameter, the GDM prevalence assumed in each study varied by area and criteria $(0.016 \sim 0.162)$. Most studies assumed universal screening uptake (100\%) for comparison. Two studies considered the real uptake and acceptance rates, ${ }^{11,40}$ while three also considered the option of screening the high-risk popula- 


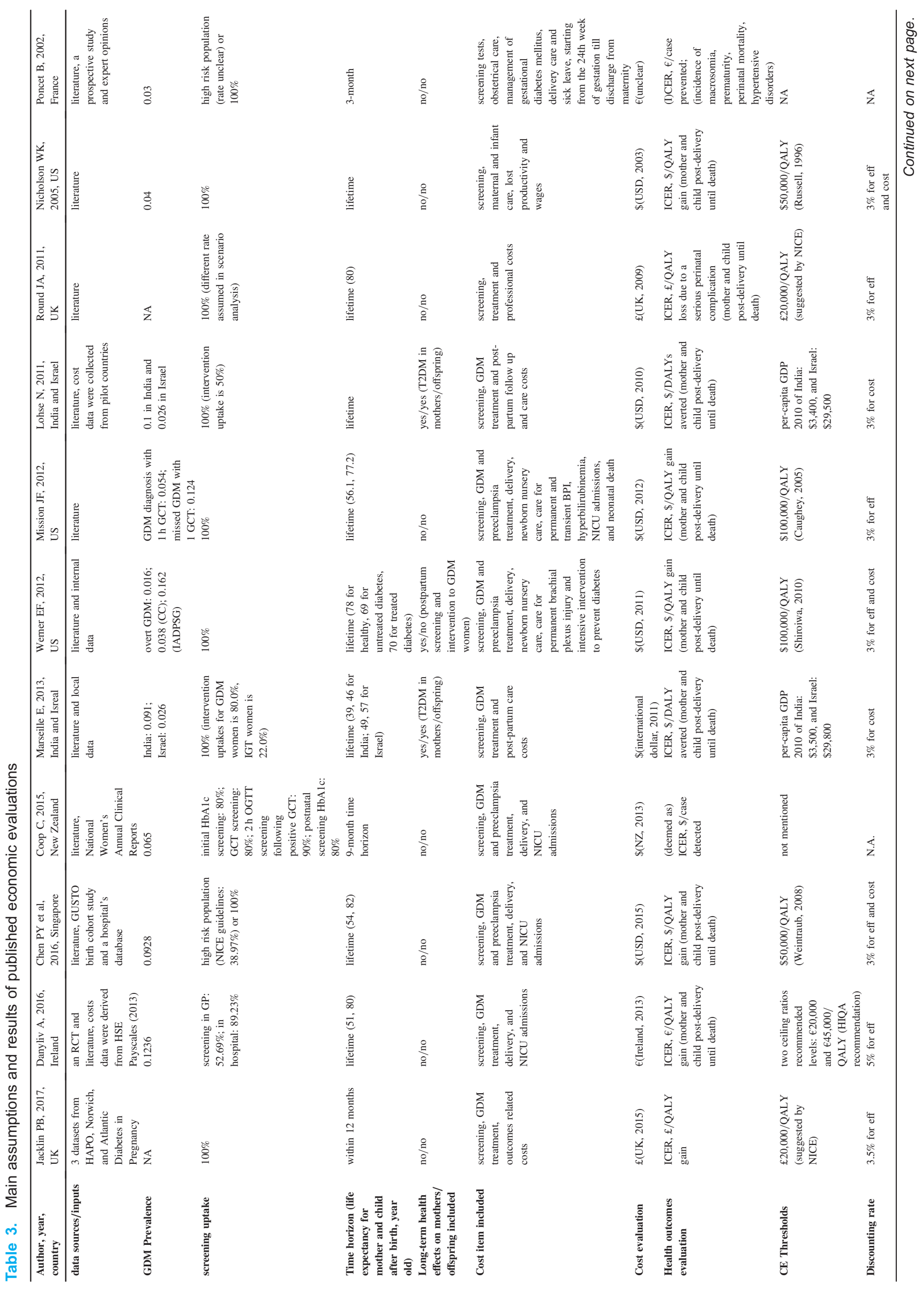




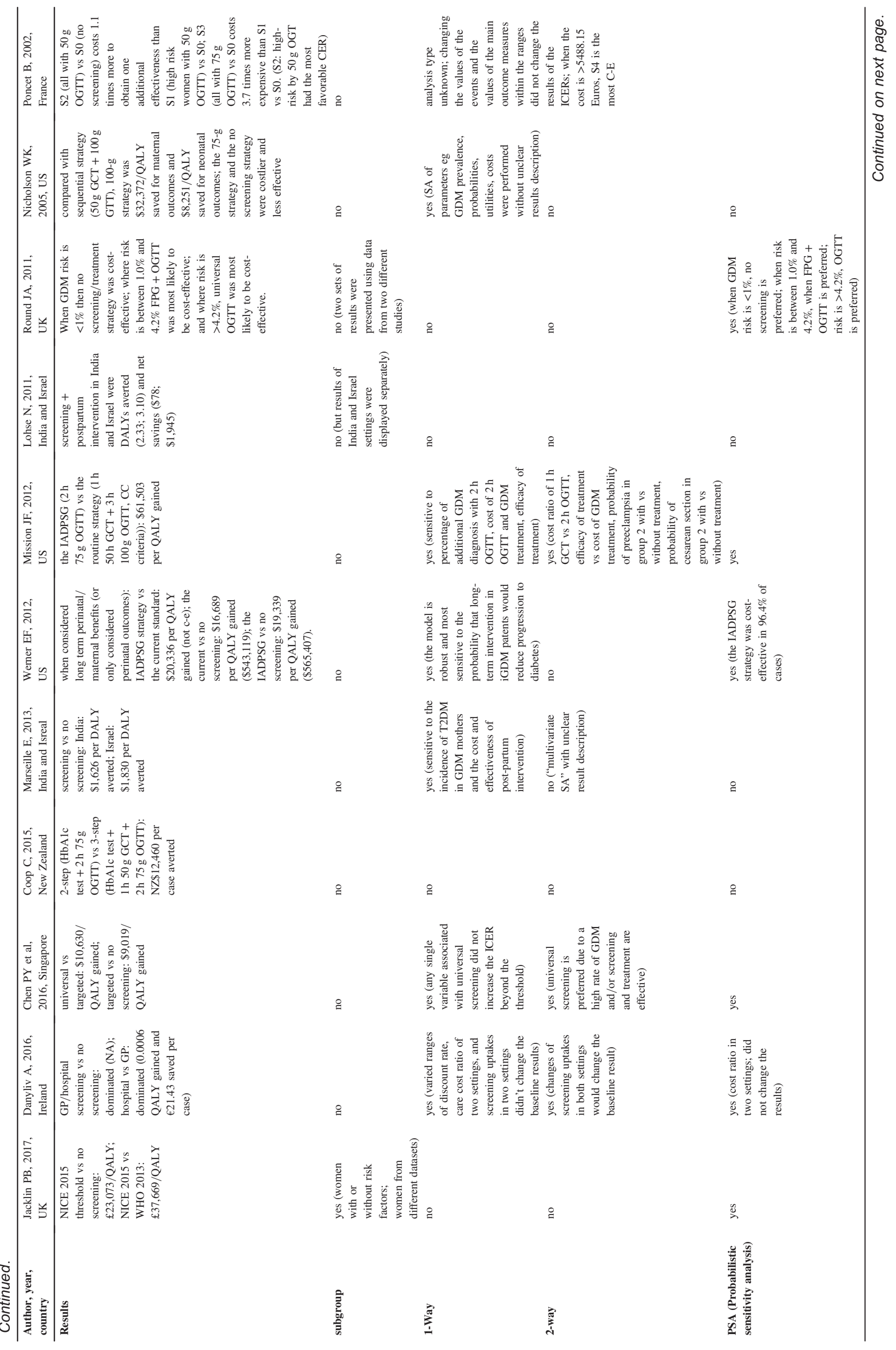




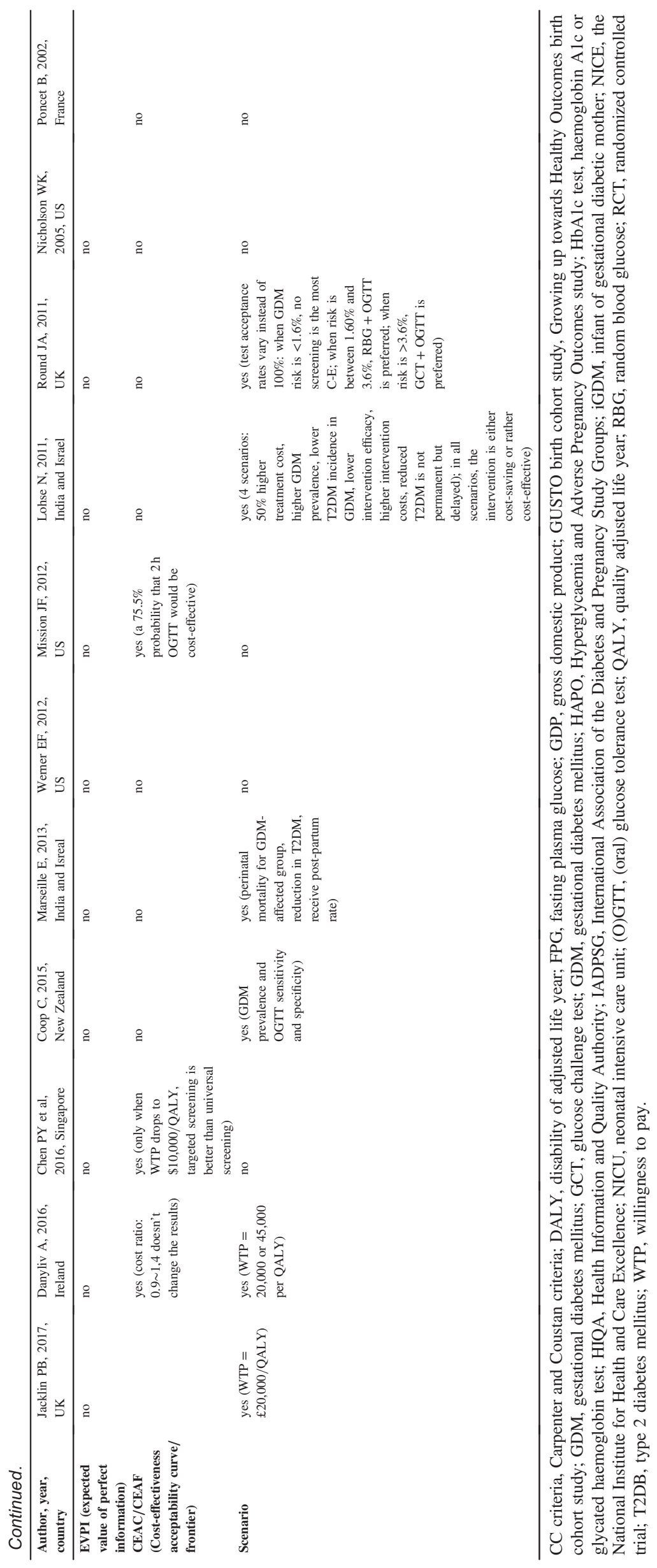


tion. ${ }^{38,39,45}$ Only one study (two articles) simulated long-term health effects on mothers and offspring. ${ }^{36,37}$ Most studies used the incremental cost-effectiveness ratio (ICER) to determine cost-effectiveness, with a diversified willingness to pay (WTP) threshold across study settings: £20,000 (suggested by NICE), ${ }^{38,43} € 20,000 / 45,000$ (Health Information and Quality Authority (HIQA) guidelines for the Republic of Ireland), ${ }^{11}$ $\$ 50,000,{ }^{39,44}$ or $\$ 100,000^{41,42}$ (commonly referenced in American studies), ${ }^{46}$ per-capita GDP. ${ }^{36,37}$ (low resource countries usually refer to this threshold). ${ }^{47}$

Compared with no screening, a screening strategy was considered dominated, ${ }^{11,44}$ cost-effective (C-E), ${ }^{37,39}$ or not C-E when the women were without risk factors (recommended by NICE; eg, polycystic ovary syndrome, previous stillbirth, or recurrent glycosuria) or when the GDM risk was less than $1 \%{ }^{38,43}$ The two-step approach described here was compared to the onestep approach ( $2 \mathrm{hr}$ OGTT at 24-28 weeks), with the execution details differing slightly among the studies $((\mathrm{HbA} 1 \mathrm{c}$ test at first booking +) $1 \mathrm{hr}$ GCT $\pm 2 / 3 \mathrm{hr}$ OGTT). Compared with the twostep approach, the IADPSG (2010) diagnostic approach (onestep) cost more, detected more cases, and proved to be C-E (under baseline consumption $)^{40,42}$ or C-E only when post-delivery care reduced diabetes incidence. ${ }^{41}$ Regarding the comparison of NICE (2015) and the IADPSG (2010) diagnostic thresholds, the lower FPG threshold of IADPSG detected more cases and was considered C-E only under a higher WTP ( $\left\{30,000\right.$ per QALY) ${ }^{38}$

The coverage of the screening program tended to influence cost-effectiveness - universal screening or options with a higher screening uptake would be more C-E or even dominated compared with the alternative of only screening the high-risk population ${ }^{41}$ or a population with a low uptake. ${ }^{11}$ The GDM risk tended to affect cost-effectiveness, as well as we mentioned earlier, among women with or without lower risk factors (recommended by NICE), no screening strategy (or strict diagnostic threshold) was likely to be C-E. ${ }^{38,43}$

Regarding uncertainties, seven studies included a one-way sensitivity analysis (SA) and three reported a two-way SA. In all, five studies presented a probabilistic SA, among which, three presented results using cost-effectiveness acceptability curves/ frontier. However, no study performed the expected value of perfect information analysis. Five studies conducted a scenario SA. Of all the existing SA parameters, the most influential ones include: the uptake of screening ${ }^{11,43}$; GDM prevalence ${ }^{38,39,43}$; effectiveness, sensitivity, and specificity of screening ${ }^{39,42}$; efficacy of treatment ${ }^{39,42}$; incidence of T2DM in GDM mothers ${ }^{37}$; cost and effectiveness of post-partum intervention ${ }^{37,41}$; cost of screening $^{42}$; cost of GDM treatment ${ }^{42}$; and $\mathrm{WTP}^{38,39}$ in the respective studies.

\section{Quality assessment and critical appraisal}

The quality scores for the 10 studies shown in Table 4 demonstrate that, on average, $81 \%(68-97 \%)$ of the items on Drummond's checklist were addressed. Specific sub-question scores are shown in eTable 2. Most studies reported problems with Questions 3, 4, and 7. In Question 3, effectiveness based on previous randomized control trials (RCTs) and/or systematic overview required clarification. However, only two studies provided details of their search strategy and the rules for inclusion or exclusion. ${ }^{41,45}$ For Question 4, due to differences in the analytical perspective, the relevant costs and consequences were varied. Only one paper mentioned both capital and operating costs. ${ }^{42}$ Regarding Question 7 , two papers did not consider long-term effectiveness, ${ }^{40,45}$ and five did not include discounting. Seven of the eleven papers scored over $80 \%$.

Table 4. Critical assessment (Methods for the Economic Evaluation of Health Care Programmes: Assessing Economic Evaluations Checklist)

\begin{tabular}{|c|c|c|c|c|c|c|c|c|c|c|c|c|c|}
\hline & Questions & $\begin{array}{c}\text { Jacklin } \\
2017\end{array}$ & $\begin{array}{c}\text { Danyliv } \\
2016\end{array}$ & $\begin{array}{l}\text { Chen } \\
2016\end{array}$ & $\begin{array}{l}\text { Coop } \\
2015\end{array}$ & $\begin{array}{l}\text { Marseille } \\
2013^{\mathrm{a}}\end{array}$ & $\begin{array}{l}\text { Werner } \\
2012\end{array}$ & $\begin{array}{c}\text { Mission } \\
2012\end{array}$ & $\begin{array}{l}\text { Lohse } \\
2011^{\text {a }}\end{array}$ & $\begin{array}{c}\text { Round } \\
2011\end{array}$ & $\begin{array}{l}\text { Nicholson } \\
2005\end{array}$ & $\begin{array}{l}\text { Poncet } \\
2002\end{array}$ & Average \\
\hline 1 & $\begin{array}{l}\text { Was a well-defined question posed } \\
\text { in answerable form? }\end{array}$ & $100 \%$ & $100 \%$ & $100 \%$ & $100 \%$ & $75 \%$ & $100 \%$ & $100 \%$ & $75 \%$ & $100 \%$ & $100 \%$ & $75 \%$ & $93 \%$ \\
\hline 2 & $\begin{array}{l}\text { Was a comprehensive description of } \\
\text { the competing alternatives given? }\end{array}$ & $100 \%$ & $100 \%$ & $100 \%$ & $100 \%$ & $100 \%$ & $100 \%$ & $100 \%$ & $100 \%$ & $100 \%$ & $100 \%$ & $100 \%$ & $100 \%$ \\
\hline 3 & $\begin{array}{l}\text { Was the effectiveness of the programmes } \\
\text { or services established? }\end{array}$ & $75 \%$ & $83 \%$ & $75 \%$ & $75 \%$ & $75 \%$ & $100 \%$ & $25 \%$ & $75 \%$ & $83 \%$ & $50 \%$ & $100 \%$ & $74 \%$ \\
\hline 4 & $\begin{array}{l}\text { Were all the important and relevant costs } \\
\text { and consequences for each alternative } \\
\text { identified? }\end{array}$ & $100 \%$ & $83 \%$ & $50 \%$ & $33 \%$ & $50 \%$ & $33 \%$ & $33 \%$ & $67 \%$ & $17 \%$ & $83 \%$ & $50 \%$ & $50 \%$ \\
\hline 5 & $\begin{array}{l}\text { Were costs and consequences measured } \\
\text { accurately in appropriate physical units } \\
\text { prior to valuation? }\end{array}$ & $100 \%$ & $100 \%$ & $100 \%$ & $100 \%$ & $100 \%$ & $100 \%$ & $100 \%$ & $100 \%$ & $100 \%$ & $100 \%$ & $100 \%$ & $100 \%$ \\
\hline 6 & $\begin{array}{l}\text { Were costs and consequences valued } \\
\text { credibly? }\end{array}$ & $100 \%$ & $100 \%$ & $100 \%$ & $100 \%$ & $100 \%$ & $100 \%$ & $100 \%$ & $75 \%$ & $100 \%$ & $75 \%$ & $100 \%$ & $95 \%$ \\
\hline 7 & $\begin{array}{l}\text { Were costs and consequences adjusted } \\
\text { for differential timing? }\end{array}$ & N.A. & $100 \%$ & $50 \%$ & N.A. & $50 \%$ & $100 \%$ & $50 \%$ & $50 \%$ & $100 \%$ & $50 \%$ & N.A. & $55 \%$ \\
\hline 8 & $\begin{array}{l}\text { Was an incremental analysis of costs and } \\
\text { consequences of alternatives performed? }\end{array}$ & $100 \%$ & $100 \%$ & $100 \%$ & $0 \%$ & $100 \%$ & $100 \%$ & $100 \%$ & $100 \%$ & $100 \%$ & $100 \%$ & $100 \%$ & $90 \%$ \\
\hline 9 & $\begin{array}{l}\text { Was uncertainty in the estimates of } \\
\text { costs and consequences adequately } \\
\text { characterized? }\end{array}$ & $100 \%$ & $100 \%$ & $83 \%$ & $50 \%$ & $83 \%$ & $100 \%$ & $83 \%$ & $25 \%$ & $100 \%$ & $0 \%$ & $17 \%$ & $64 \%$ \\
\hline \multirow[t]{2}{*}{10} & $\begin{array}{l}\text { Did the presentation and discussion } \\
\text { of study results include all issues of } \\
\text { concern to users? }\end{array}$ & $100 \%$ & $100 \%$ & $100 \%$ & $100 \%$ & $83 \%$ & $75 \%$ & $67 \%$ & $33 \%$ & $83 \%$ & $17 \%$ & $83 \%$ & $74 \%$ \\
\hline & Average & $96 \%$ & $97 \%$ & $86 \%$ & $73 \%$ & $82 \%$ & $91 \%$ & $76 \%$ & $70 \%$ & $88 \%$ & $68 \%$ & $81 \%$ & $81 \%$ \\
\hline
\end{tabular}

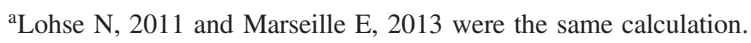




\section{DISCUSSION}

We reviewed the published economic evaluations of GDM screening and assessed quality in terms of options design, modelling, results, and parameters for sensitivity analysis for each paper, which were different from each other. Overall, screening is $\mathrm{C}-\mathrm{E}$ or even dominant over no screening. Although the dominance of specific screening methods or targets could not be determined, recent studies have focused on screening using the $2 \mathrm{hr} 75 \mathrm{~g}$ OGTT (IADPSG criteria) and compared it with no screening $11,36,37,39$ or with status quo (the two-step strategy). ${ }^{40-42}$ In the end, the method that results in more cases detected is likely to be C-E compared to the alternative on the conditions that postnatal care reduces diabetes incidence and that WTP increases. The results show that the one-step screening is comparatively more C-E than the two-step ${ }^{41,42}$ and the two-step is more C-E than the three-step. ${ }^{40}$ With a higher WTP, the option with a low diagnostic threshold (eg, the IADPSG criteria) is more C-E than its counterpart (eg, NICE 2015). ${ }^{38} \mathrm{~A}$ universal screening is C-E or dominant over no screening or a screening targeting the high-risk population (NICE), ${ }^{11,41}$ where a relatively large proportion of cases were detected. Conversely, the results of economic evaluation are different when targeting low-risk population. ${ }^{38,43}$ The dominance largely depends on the risks of the target individuals and the acceptability of the screening options. ${ }^{38,43}$

Other than the screening protocols and diagnosis criteria under different healthcare systems, and epidemiological characteristics of GDM (GDM prevalence and mortality) in the target population, other key factors that affect cost-effectiveness of the screening include: detection efficacy, ${ }^{42}$ long-term benefits attributable to early detection, ${ }^{41}$ treatment efficacy, ${ }^{42}$ and the cost of screening. ${ }^{42}$ In particular, the consideration of long-term outcomes has a significant influence on the results, ${ }^{41}$ which were not considered in almost all the studies examined, implying the importance of implementing effective postnatal interventions.

None of the studies compared different screening timings. Screening is usually performed at $24-28$ weeks. Recent studies have suggested that GDM screening occur in the first trimester, accompanying other regular tests assessing a combination of maternal characteristics and biomarkers, ${ }^{4-50}$ since a previous study suggested that first-trimester $\mathrm{HbA1c}$ alone does not have sufficient sensitivity or specificity for diagnosis. ${ }^{51}$ Moreover, most studies were conducted in developed countries and evidence from low-income and middle-income countries is lacking.

Our review identified some methodological inconsistencies. For example, the difference between "ICER" and "CER," definition of the C-E threshold, and discount rate were not clarified. ${ }^{40}$ Utilities and treatment effects were not clearly described either. ${ }^{45}$ While the type of SA is not considered in the quality assessment ( 99 in the uncertainty analysis), most studies conducted a deterministic and not a probabilistic SA, even though the latter can assess the cost-effectiveness of an target option at a certain threshold ${ }^{52}$ and characterize the combined effects of all parameter uncertainties simultaneously. ${ }^{10}$

Our review also identified a lack of clarity in the analytical perspective, types of study design, health gains, consideration of uncertainties, and discounting in some existing studies, which if included, would have made the results more reliable. ${ }^{10}$ Regarding reporting standards, the newly-launched guidelines for economic evaluation, such as the Consolidated Health Economic Evaluation Reporting Standards (CHEERS) statement, ${ }^{53}$ methodological guidelines proposed by NICE from the United Kingdom, ${ }^{54}$ and the International Society for Pharmacoeconomics and Outcomes Research, ${ }^{55}$ facilitate the creation of high-quality evidence.

\section{Conclusions}

Our review shows that the screening program for GDM during pregnancy is C-E in general. The one-step screening, with more cases detected, is more likely to be C-E than the two-step screening. Universal screening is more likely to be C-E than screening targeting high-risk population. A higher screening uptake, more effective treatment, and postnatal interventions contribute toward improving cost-effectiveness. The quality assessment identified several weaknesses in performing and reporting economic evaluations and leaves us with lessons and research tasks for the future.

\section{ACKNOWLEDGEMENTS}

We thank Dr Agari Takahiro and Ms Naito Yumi for the help of critical assessment of the first one economics evaluation. This study is supported by JSPS KAKENHI Grant Number 18J22137. The funders did not have any role in the study design, data collection, and analysis, interpretation of data or in writing the manuscript.

Contribution to authorship: Xiuting Mo and Ruoyan Gai Tobe conceived, planned and carried out the review. Xiuting Mo performed the analyses and drafted the manuscript. Xiuting Mo, Ruoyan Gai Tobe, Yoshimitsu Takahashi, Naoko Arata, Tippawan Liabsuetrakul, Takeo Nakayama, and Mori Rintaro revised and accepted the final manuscript.

Conflicts of interest: None declared.

\section{APPENDIX A. SUPPLEMENTARY DATA}

Supplementary data related to this article can be found at https:// doi.org/10.2188/jea.JE20190338.

\section{REFERENCES}

1. Sacks DA, Hadden DR, Maresh M, et al; HAPO Study Cooperative Research Group. Frequency of gestational diabetes mellitus at collaborating centers based on IADPSG consensus panel-recommended criteria: the Hyperglycemia and Adverse Pregnancy Outcome (HAPO) Study. Diabetes Care. 2012;35(3):526-528.

2. Caissutti C, Berghella V. Scientific evidence for different options for GDM screening and management: controversies and review of the literature. BioMed Res Int. 2017;2017:2746471.

3. Ziegler AG, Wallner M, Kaiser I, et al. Long-term protective effect of lactation on the development of type 2 diabetes in women with recent gestational diabetes mellitus. Diabetes. 2012;61(12):31673171.

4. Simeoni U, Barker DJ. Offspring of diabetic pregnancy: long-term outcomes. Paper presented at: Seminars in Fetal and Neonatal Medicine, 2009.

5. Mitanchez D, Burguet A, Simeoni U. Infants born to mothers with gestational diabetes mellitus: mild neonatal effects, a long-term threat to global health. J Pediatr. 2014;164(3):445-450.

6. Fitria N, van Asselt ADI, Postma MJ. Cost-effectiveness of controlling gestational diabetes mellitus: a systematic review. Eur J Health Econ. 2019;20(3):407-417.

7. Werbrouck A, Schmidt M, Putman K, et al. A systematic review on costs and cost-effectiveness of screening and prevention of type 2 diabetes in women with prior gestational diabetes: exploring uncharted territory. Diabetes Res Clin Pract. 2019;147:138-148. 
8. Poolsup N, Suksomboon N, Amin M. Effect of treatment of gestational diabetes mellitus: a systematic review and meta-analysis. PLoS One. 2014;9(3):e92485.

9. Vogel N, Burnand B, Vial Y, Ruiz J, Paccaud F, Hohlfeld P. Screening for gestational diabetes: variation in guidelines. Eur $J$ Obstet Gynecol Reprod Biol. 2000;91(1):29-36.

10. Drummond MF, Sculpher MJ, Claxton K, Stoddart GL, Torrance GW. Methods for the economic evaluation of health care programmes. Oxford University Press; 2015.

11. Danyliv A, Gillespie P, O'Neill C, et al. The cost-effectiveness of screening for gestational diabetes mellitus in primary and secondary care in the Republic of Ireland. Diabetologia. 2016;59(3):436-444.

12. Liang SQ, He ZL, Tang Y, et al. PDB58 gestational diabetes screening in China: a cost-effectiveness study. Value Health. 2019; 22:S150.

13. Duarte A, Griffin S, Sheldon T, et al. Identification and treatment of gestational diabetes: an economic evaluation. BJOG. 2018;125:112113

14. Li LJ, Aris IM, Chong Y, Gluckman P, Ang S, Tan K. Comparison of diagnostic value and cost-effectiveness in screening approaches for postpartum pre-diabetes and diabetes among women with history of gestational diabetes. Diabetologia. 2018;61:S458.

15. Walker AR, Valent A, Caughey AB. 494: Positivity thresholds of $\mathrm{HbAlc}$ assay as a screening test for gestational diabetes mellitus in the first trimester in high-risk populations. Am J Obstet Gynecol. 2017;216(1):S291-S292.

16. Pearson LJ, Mission JF, Niu B, Caughey AB. 297: Gestational diabetes screening with the IADPSG guidelines: a cost-effectiveness analysis by race/ethnicity. Am J Obstet Gynecol. 2016;214(1):S170S171.

17. Ming WK. PDB14 - Gestational diabetes screening for Chinese women: a cost-effectiveness analysis. Value Health. 2016;19(7): A897.

18. Zhang L, Chen W, Wang Y, Ma R, Du M, Xu X. PDB60 - Costeffectiveness analysis of gestational diabetes mellitus screening in urban Chinese setting. Value Health. 2015;18(7):A607-A608.

19. Quitian H, Acosta A, Martinez RA, et al. PDB75 - Economic evaluation of the one-step scheme compared with two-steps for screening and diagnosis of gestational diabetes in Colombia. Value Health. 2015;18(7):A610.

20. Chen PY, Finkelstein EA, Kruger E, Ng MJ, Tan KH. Costeffectiveness analysis on gestational diabetes mellitus screening strategies. J Matern Fetal Neonatal Med. 2014;27(Suppl 1):69-70.

21. Werner EF, Pettker CM, Reel M, Zuckerwise LC, Funai EF, Thung SF. 250: Long term diabetes risk reduction necessary for gestational diabetes screening to be cost effective. Am J Obstet Gynecol. 2012; 206(1):S122-S123.

22. Reel M, Werner E, Pettker C, Funai E, Thung S. 280: Screening for gestational diabetes with a 1 hour glucose challenge test: is a 130 $\mathrm{mg} / \mathrm{dL}$ threshold more cost-effective than a $140 \mathrm{mg} / \mathrm{dL}$ threshold? Am J Obstet Gynecol. 2011;204(1):S117-S118.

23. Van Leeuwen M, Vijgen S, Opmeer BC, Evers I, Mol BW. 264: Cost-effectiveness analysis of screening for GDM. Am J Obstet Gynecol. 2009;201(6):S109.

24. Lee S, Pettker C, Funai E, Norwitz E, Thung S. 694: Is lowering the diagnostic threshold for gestational diabetes (GDM) cost-effective? Implications from the hyperglycemia and adverse pregnancy outcomes (HAPO) trial. Am J Obstet Gynecol. 2008;199(6):S199.

25. Thung S, Pettker C, Funai E. 355: Screening for gestational diabetes: Is a $130 \mathrm{mg} / \mathrm{dL}$ or $140 \mathrm{mg} / \mathrm{dL}$ glucose challenge test threshold more cost-effective? Am J Obstet Gynecol. 2007;197(6):S109.

26. Cade TJ, Polyakov A, Brennecke SP. Implications of the introduction of new criteria for the diagnosis of gestational diabetes: a health outcome and cost of care analysis. BMJ Open. 2019;9(1): e023293.

27. Sorts $\emptyset$ C, Komkova A, Sandbæk A, et al. Effect of screening for type 2 diabetes on healthcare costs: a register-based study among 139,075 individuals diagnosed with diabetes in Denmark between 2001 and 2009. Diabetologia. 2018;61(6):1306-1314.

28. Rodríguez-Pérez MC, Orozco-Beltrán D, Gil-Guillén V, et al.
Clinical applicability and cost-effectiveness of DIABSCORE in screening for type 2 diabetes in primary care. Diabetes Res Clin Pract. 2017;130:15-23

29. Duran A, Saenz S, Torrejon MJ, et al. IADPSG criteria for screening and diagnosis of gestational diabetes mellitus (GDM) induce better outcomes of pregnancy with a lower cost in a large cohort of pregnant women. Diabetes. 2014;63:A338-A339.

30. Gillespie P, O’Neill C, Avalos G, Dunne FP; ALANTIC DIP Collaborators. New estimates of the costs of universal screening for gestational diabetes mellitus in Ireland. Ir Med J. 2012;105(5 Suppl): $15-18$.

31. Ayach W, Costa RA, Calderon Ide M, Rudge MV. Comparison between 100-g glucose tolerance test and two other screening tests for gestational diabetes: combined fasting glucose with risk factors and 50-g glucose tolerance test (Provisional abstract). Sao Paulo Med J. 2006;124(1):4-9.

32. Rey E, Hudon L, Michon N, Boucher P, Ethier J, Saint-Louis P. Fasting plasma glucose versus glucose challenge test: screening for gestational diabetes and cost effectiveness. Clin Biochem. 2004;37(9): 780-784.

33. Larijani B, Hossein-Nezhad A, Vassigh AR. Effect of varying threshold and selective versus universal strategies on the cost in gestational diabetes mellitus. Arch Iran Med. 2004;7(4):267-271.

34. Di Cianni G, Volpe L, Casadidio I, et al. Universal screening and intensive metabolic management of gestational diabetes: costeffectiveness in Italy. Acta Diabetol. 2002;39(2):69-73.

35. Weiner CP, Fraser MM, Burns JM, Schnoor D, Herrig J, Whitaker LA. Cost efficacy of routine screening for diabetes in pregnancy: 1-h versus 2-h specimen. Diabetes Care. 1986;9(3):255-259.

36. Lohse N, Marseille E, Kahn JG. Development of a model to assess the cost-effectiveness of gestational diabetes mellitus screening and lifestyle change for the prevention of type 2 diabetes mellitus. Int $J$ Gynaecol Obstet. 2011;115(Suppl 1):S20-S25.

37. Marseille E, Lohse N, Jiwani A, et al. The cost-effectiveness of gestational diabetes screening including prevention of type 2 diabetes: application of a new model in India and Israel. $J$ Matern Fetal Neonatal Med. 2013;26(8):802-810.

38. Jacklin PB, Maresh MJ, Patterson CC, et al. A cost-effectiveness comparison of the NICE 2015 and WHO 2013 diagnostic criteria for women with gestational diabetes with and without risk factors. $B M J$ Open. 2017;7(8):e016621.

39. Chen PY, Finkelstein EA, Ng MJ, et al. Incremental costeffectiveness analysis of gestational diabetes mellitus screening strategies in Singapore. Asia Pac J Public Health. 2016;28(1):15-25.

40. Coop C, Edlin R, Brown J, Farquhar C. Cost-effectiveness of the New Zealand diabetes in pregnancy guideline screening recommendations. BMJ Open. 2015;5(6):e006996.

41. Werner EF, Pettker CM, Zuckerwise L, et al. Screening for gestational diabetes mellitus: are the criteria proposed by the international association of the Diabetes and Pregnancy Study Groups cost-effective? Diabetes Care. 2012;35(3):529-535.

42. Mission J, Ohno M, Yanit K, Cheng Y, Caughey A. Gestational diabetes screening with the new IADPSG 2 hour glucose tolerance test vs the 1 hour glucose challenge test: a cost-effectiveness analysis. Am J Obstet Gynecol. 2012;206(1):S126.

43. Round JA, Jacklin P, Fraser RB, Hughes RG, Mugglestone MA, Holt RIG. Screening for gestational diabetes mellitus: cost-utility of different screening strategies based on a woman's individual risk of disease. Diabetologia. 2011;54(2):256-263.

44. Nicholson WK, Fleisher LA, Fox HE, Powe NR. Screening for gestational diabetes mellitus: a decision and cost-effectiveness analysis of four screening strategies. Diabetes Care. 2005;28(6):1482-1484.

45. Poncet B, Touzet S, Rocher L, Berland M, Orgiazzi J, Colin C. Costeffectiveness analysis of gestational diabetes mellitus screening in France. Eur J Obstet Gynecol Reprod Biol. 2002;103(2):122-129.

46. Neumann PJ, Cohen JT, Weinstein MC. Updating cost-effectiveness -the curious resilience of the \$50,000-per-QALY threshold. $N$ Engl J Med. 2014;371(9):796-797.

47. Marseille E, Larson B, Kazi DS, Kahn JG, Rosen S. Thresholds for the cost-effectiveness of interventions: alternative approaches. Bull 
World Health Organ. 2015;93(2):118-124.

48. Nanda S, Savvidou M, Syngelaki A, Akolekar R, Nicolaides KH. Prediction of gestational diabetes mellitus by maternal factors and biomarkers at 11 to 13 weeks. Prenat Diagn. 2011;31(2):135-141.

49. Syngelaki A, Pastides A, Kotecha R, Wright A, Akolekar R, Nicolaides KH. First-trimester screening for gestational diabetes mellitus based on maternal characteristics and history. Fetal Diagn Ther. 2015;38(1):14-21.

50. Maegawa Y, Sugiyama T, Kusaka H, Mitao M, Toyoda N. Screening tests for gestational diabetes in Japan in the 1st and 2nd trimester of pregnancy. Diabetes Res Clin Pract. 2003;62(1):47-53.

51. Benaiges D, Flores-Le Roux JA, Marcelo I, et al. Is first-trimester HbA1c useful in the diagnosis of gestational diabetes? Diabetes Res
Clin Pract. 2017:133:85-91.

52. Adalsteinsson E, Toumi M. Benefits of probabilistic sensitivity analysis - a review of NICE decisions. J Mark Access Health Policy. $2013 ; 1$.

53. Husereau D, Drummond M, Petrou S, et al; CHEERS Task Force. Consolidated Health Economic Evaluation Reporting Standards (CHEERS) statement. BMC Med. 2013;11:80.

54. Excellence NIfC. Guide to the methods of technology appraisal 2013 https://www.nice.org.uk/process/pmg9/chapter/foreword. 2013.

55. Eldessouki R, Dix Smith M. Health care system information sharing: a step toward better health globally. Value Health Reg Issues. 2012; $1(1): 118-120$ 